\title{
Linear Functional Graphs - a Data Arrangement and Visualization Tool for Linear Algebra
}

\author{
Pēteris Daugulis ${ }^{1} \mathrm{PhD}$; Anita Sondore ${ }^{2}$ Dr. math. \\ Daugavpils University, Latvia \\ peteris.daugulis@du.lv ${ }^{1}$; anita.sondore@du.lv²
}

\begin{abstract}
Visualization and mappings of mathematical data and transformations to geometric objects are important tools in any area of mathematics. Directed graphs have been used in linear algebra since D. König in the early 20th century. In this paper, we aim to give interpretations of such graphs and related data arrangements which project linear algebra to graph theory - definitions of linear functional graphs. The results were obtained by applying discrete modelling techniques to objects of linear algebra. Linear functional graphs can be used in linear algebra courses to teach the theory of linear mappings, matrix multiplication and determinants. This technique can make linear algebra learning visual and suitable for some learners.
\end{abstract}

Keywords: mathematical education, university education, visualization, linear mapping, matrix, directed graph.

\section{Introduction}

\section{Background}

University educators in engineering and most sciences must constantly work on improving the educational process and content for all levels and ages (Eurydice Brief, Modernisation..., 2014). An important study discipline requiring continuous methodological effort is mathematics (PISA 2021 Mathematics..., 2021; Zeidmane, Sergejeva, 2013; Zeidmane, Rubina, 2017; Zeidmane, Rubina, 2018; $\mathrm{Li}$, Schoenfeld, 2019). Its course concepts, methods and teaching processes must be updated and made effective (Kopeika, Zvirgzdina, 2020; Sergejeva, Aboltins, 2020).). Diversification of arrangements of numerical data (Daugulis et al., 2020) and visualization of mathematical objects are important educational and practical techniques. Such techniques are often related to encoding and coordinatization of mathematical objects, projection of derived mathematical objects to more fundamental ones. Many areas of mathematics have developed suitable visual models of objects and transformations using Euclidean geometry and graphs. The Euler-Venn diagrams and functional graphs can be mentioned as simple well-known examples.

An important branch of mathematics is linear algebra. It is a major practical tool used to model and solve engineering problems in all areas and scales from purely technical to social ones.

EXAMPLE 1. The example given by P. Daugulis (2012) is used. Suppose you are given a set of workers $A=\left\{a_{1}, \ldots, a_{m}\right\}$ and a set of tasks $B=\left\{b_{1}, \ldots, b_{n}\right\}$. Suppose each worker has performed each task and the performance time has been measured. Denote by $t_{i j}$ the time in which the worker $a_{j}$ has performed the task $b_{i}$. See also REMARK 2. One can pose the problem of comparing (rating) relative difficulty of the tasks and the relative task performance speed of the workers. A simple way would be to compare the time sums or averages $\overline{a_{j}}$ and $\overline{b_{l}}$ between objects of the same type (workers or tasks). This may fail to distinguish objects, especially if time takes values in a small discrete set (e.g., the number of working days). The authors can refine the rating system. Let us try to define a rating system for workers and tasks according to the following assumptions. The task ratings are linear with respect to the worker ratings and vice versa. It can be assumed that the contribution of $a_{j}$ to the rating $b_{i}$ is proportional to the rating of $a_{j}$ and $t_{i j}$ (since harder tasks will have larger performing times), the contribution of $b_{i}$ to the rating of $a_{j}$ is proportional to the rating of $b_{i}$ and $\frac{1}{t_{i j}}$ (since better workers will have smaller performance times). 
The authors define two normed rating vectors $\boldsymbol{a}=\left[A_{1}, \ldots, A_{m}\right]^{T}$ and $\boldsymbol{b}=\left[B_{1}, \ldots, B_{n}\right]^{T}$ which satisfy the system of equations (Formula 1):

$$
\left\{\begin{array} { l } 
{ B _ { i } = \lambda \sum _ { j = 1 } ^ { m } t _ { i j } A _ { j } , } \\
{ A _ { i } = \mu \sum _ { j = 1 } ^ { n } \frac { 1 } { t _ { j i } } B _ { j } , } \\
{ \| \boldsymbol { a } \| = \| \boldsymbol { b } \| = 1 , }
\end{array} \quad \left\{\begin{array}{c}
\boldsymbol{b}=\lambda T \boldsymbol{a} \\
\boldsymbol{a}=\mu T^{\prime} \boldsymbol{b} ; \\
\|\boldsymbol{a}\|=\|\boldsymbol{b}\|=1,
\end{array}\right.\right.
$$

where $A_{\mathrm{i}}$ and $B_{\mathrm{i}}$ are the "ratings" of the worker $a_{i}$ and the task $b_{i}$, respectively, and the linear mappings $T$ and $T$ ' are defined by the system of equations (Formula 2 ).

$$
\left\{\begin{array}{l}
T=\left[t_{i j}\right]_{n, m} ; \\
T^{\prime}=\left[\frac{1}{t_{i j}}\right]_{m, n} .
\end{array}\right.
$$

Solving the system (1), the authors get rating lists of workers and tasks. Such lists may be an important asset for managing workgroups and businesses. It is crucial to understand the linear mappings $T$ and $T$ '. This example shows the importance of linear mappings in applications.

In this article, our main goal is to find and describe effective and visual discrete models of linear mappings. This will contribute to improving the data arrangement and visualization in linear algebra. The authors describe a generalization of functional graphs for linear mappings - linear functional graphs (LFG).

Linear mappings are uniquely determined by images of elements of a basis. Therefore, linear mappings can be encoded using bases as vertex sets, while edge sets encode images of elements of bases under linear mappings. This generalization of functional graphs is similar to graphs of mappings (multivalued functions). Composition of linear mappings, matrix multiplication and determinants can be interpreted and defined in terms of LFG.

D. König (1916) seems to have been the first to describe the usage of graphs in linear algebra in the beginning of the 20th century. A modern treatment is given by R.A. Brualdi and D. Cvetkovic (2009). Vertex sets of graphs built from matrices are usually just identified with matrix rows and columns. Applications of linear algebra in graph theory appear in publications by M. Doob (1984), E.A. Kalinina and G.M. Khitrov (2017).

Matrix graphs and their full interpretations are not widely used in linear algebra textbooks, one of our goals is to popularize them. In this article, the authors define two versions of LFG depending on the number of bases necessary to define the linear mapping. The authors interpret matrix multiplication and determinants using LFG. These results were essentially described by P. Daugulis (1998).

The authors consider finite-dimensional $k$-linear spaces over some fixed field $k$. Matrices are denoted using bold letters. The authors denote a linear space $L$ with an ordered basis $B_{L}$ as $\left(L, B_{L}\right)$.

If $B_{L}=\left[e_{1}, \ldots, e_{n}\right]$ is an ordered basis of $L$ and $a \in L$, then the coordinate vector (column) of $a$ with respect to $B_{L}$ is denoted by $[a]_{B_{L}}$.

An edge-weighted directed graph (digraph) is a triple $\Gamma=(V, E, w)$, where

$V$ is the set of vertices;

$E \subseteq V \times V$ is the set of edges, $\left(v, v^{\prime}\right) \in E$ means the directed edge from $v^{\prime} \in V$ to $v \in V$;

$w: V \times V \rightarrow k$ is the edge-weight function, $w\left(v, v^{\prime}\right)=0$ if and only if $\left(v, v^{\prime}\right) \notin E$. The edge corresponding to $w\left(v, v^{\prime}\right)$ is $v^{\prime} \stackrel{w\left(v, v^{\prime}\right)}{\longrightarrow} v$.

The authors denote by $(V, E)$ a digraph for which the weight function is in some sense trivial or is not used.

\section{Review of functional graphs}

The authors give this review for notational purposes. 
A mapping (multivalued function) $f: A \rightarrow B$ from a set $A$ (domain) to a set $B$ (range) is a function $A \rightarrow 2^{B}$ (the power set of $B$ ). In the special case when $|f(a)|=1$ for every $a \in A$ the authors identify $f$ with the function $A \rightarrow B$.

Suppose the sets $A, B$ and a mapping $f: A \rightarrow B$ are given. Define the 2-functional graph (2-FG) of $f$ as the bipartite digraph $\Gamma(A, B, f)=\left(A \cup B, E_{f}\right)$, where the set of edges $E_{f}=\amalg_{a \in A} \amalg_{b \in f(a)}(b, a)$. Given a set $A$ and an endomapping $f: A \rightarrow A$ define the 1-functional graph (1-FG) of $f$ as $\Gamma(A, f)=\left(A, E_{f}\right)$, where $E_{f}=\bigsqcup_{a \in A} \amalg_{a, \in f(a)}\left(a^{\prime}, a\right)$.

EXAMPLE 2. Let $A=\{1,2,3\}$ and $B=\{a, b, c\}$. Define a mapping $h: A \rightarrow B$ by setting $h(1)=\{a, b\}, h(2)=a$, $h(3)=\{a, c\}$. The 2-FG $\Gamma(A, B, h)$ is shown in Figure 1 (left).

EXAMPLE 3. Let A be as in the previous example. Define a function $f: A \rightarrow A$ by setting $f(1)=2, f(2)=3$, $f(3)=1$. The 1-FG $\Gamma(A, f)$ is shown in Figure 1 (right).
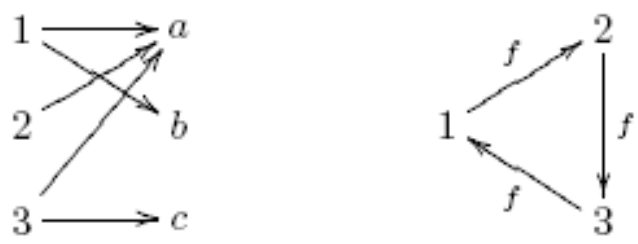

Figure 1. The 2-FG for the mapping $h$ of Example 2 and the 1-FG for the mapping $f$ of Example 3.

The aim of the paper is to give interpretations of such graphs and related data arrangements which project linear algebra to graph theory - definitions of linear functional graphs.

\section{Methodology}

The main research task was to generalize functional graphs in the linear algebra setting to be used in teaching linear algebra to rigorously interpret the known graphical techniques in linear algebra. The described discrete models were developed after an analysis of existing literature. Related methodological results were obtained applying theoretical results in linear algebra courses, various qualitative research methods (Mārtinsone et al., 2016). Discrete modelling methods were used. Some additional results were obtained and approbated by the authors teaching university-level linear algebra courses at Daugavpils University over 10 years.

\section{Results and Discussion}

\section{Linear functional graphs}

In this section, the authors describe generalizations of functional graphs for linear mappings. Edgeweighted digraphs are assigned to general matrices in the same way as unweighted digraphs are assigned to binary matrices. A suitable eponym-free name for them would be linear functional graphs $(L F G)$. Two separate cases must be considered: linear mappings between two spaces and linear operators.

Linear mappings are functions between linear spaces which preserve linear operations. Let $L, U$ be $k$-linear spaces. A function $f: L \rightarrow U$ is a linear mapping if

$$
f\left(l_{1}+l_{2}\right)=f\left(l_{1}\right)+f\left(l_{2}\right) \text { for all } l_{1}, l_{2} \in L
$$

$f(\lambda l)=\lambda f(l)$ for all $l \in L$ and $\lambda \in k$.

$\operatorname{Hom}(L, U)$ - the space of all linear mappings from $L$ to $U$.

Most functions studied and used in linear algebra are linear mappings. Linear mappings are also useful in practical applications. For example, many important geometrical transformations are linear mappings (Blyth, Robertson, 2002).

The modelling idea. Here is an attempt to generalize the functional graphs for linear mappings.

The first step is to find objects in linear spaces which should be modelled as graph vertices. Elements of linear spaces are uniquely expressed as linear combinations of elements of their bases. This observation leads to the idea to start by choosing bases as vertex sets. Linear mappings are uniquely 
determined by images of elements of a basis of the domain space. These images are, in turn, uniquely expressed as linear combinations of elements of a basis of the range space. Therefore, a linear mapping can be uniquely encoded using edge-weighted directed graphs:

- the vertex set is the union of bases or the basis;

- the set of weighted edges is determined by the images of basis elements under linear mappings: if $f(e)$ contains a summand $f_{i} t_{i}$, where $t_{i}$ is an element of a basis, then there is an arrow from $e$ to $t_{i}$ with weight $f_{i}$.

The generalization of functional graphs for linear mappings (linear functional graphs, LFG) is similar to functional graphs of multivalued functions.

Graphs for linear mappings between two spaces

Let $\left(L, B_{L}\right),\left(U, B_{U}\right)$ be linear spaces, $B_{L}=\left[e_{1}, \ldots, e_{n}\right]$ and $B_{U}=\left[t_{1}, \ldots, t_{m}\right]$ - ordered bases. Let $f \in \operatorname{Hom}(L, U)$ be defined by linear combination (Formula 3 ).

$$
f\left(e_{j}\right):=\sum_{i=1}^{m} f_{i j} t_{i}
$$

The matrix $\left[f_{i j}\right]$ - the $f$-matrix with respect to $B_{L}$ and $B_{U}$ is denoted by $[f]_{B_{L}, B_{U}}$ or $\boldsymbol{F}$.

DEFINITION 1. Define an edge-weighted digraph $\Lambda\left(B_{L}, B_{U}, f\right)=\Lambda\left(B_{L}, B_{U}, \boldsymbol{F}\right):=\left(B_{L} \cup B_{U}, E, w_{f}\right)$, where $E=\bigsqcup_{i, j: f_{i j} \neq 0}\left(t_{i}, e_{j}\right), w_{f}\left(t_{i}, e_{j}\right):=f_{i j} . \Lambda\left(B_{L}, B_{U}, f\right)=\Lambda\left(B_{L}, B_{U}, \boldsymbol{F}\right)$ is called the 2-LFG of $f$ or $\boldsymbol{F}$ with respect to $B_{L}, B_{U}$.

REMARK 1. 2-LFG are bipartite graphs. If the weight of an edge is 1, then it is not shown as an edge weight. Thus unweighted graphs correspond to binary matrices.

EXAMPLE 4. Let $\left(L,\left[e_{1}, e_{2}, e_{3}, e_{4}\right]\right),\left(U,\left[t_{1}, t_{2}\right]\right)$ be linear spaces. The 2-LFG for the linear mapping $f: L \rightarrow U$ corresponding to the matrix $\left[\begin{array}{l}\lambda 000 \\ 1 \lambda 10\end{array}\right]$ is shown in Figure 2.

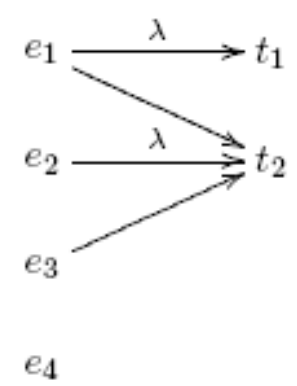

Figure 2. The 2-LFG for the linear mapping of Example 4.

REMARK 2. A 2-LFG graph for Example 1 is shown in Figure 3.

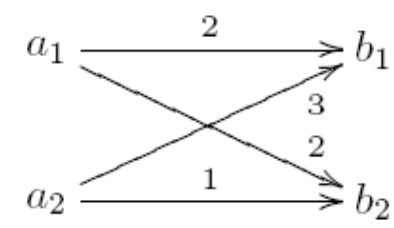

Figure 3. A 2-LFG for Example 1.

\section{Matrix multiplication using 2-LFG}

2-LFG allows us to interpret matrix multiplication in a graph-theoretic way (Brualdi, Cvetkovic, 2009).

Suppose that a $m \times n$ matrix $\boldsymbol{A}=\left[a_{i j}\right]$, and a $n \times r$ matrix $\boldsymbol{B}=\left[b_{i j}\right]$ are given. They can be viewed as matrices of linear mappings. Let $f \in \operatorname{Hom}(V, U)$ and $g \in \operatorname{Hom}(L, V)$ for some linear spaces $\left(L, B_{L}\right)$, $\left(V, B_{V}\right)$ and $\left(U, B_{U}\right)$ with respect to the given bases: $\boldsymbol{A}=[f]_{B_{V}, B_{U}}, \boldsymbol{B}=[g]_{B_{L}, B_{V}}$. The authors construct the corresponding 2-LFGs $\Lambda\left(B_{V}, B_{U}, \boldsymbol{A}\right)$ and $\Lambda\left(B_{L}, B_{V}, \boldsymbol{B}\right)$. The matrix product $\boldsymbol{A} \boldsymbol{B}=\boldsymbol{C}=\left[c_{i j}\right]$ is equal to $[f \circ g]_{B_{L}, B_{U}}$. It is seen that $c_{i j}$ is equal to the sum of products of edge weights for paths from the jth 
vertex of $B_{L}$ to the ith vertex of $B_{U}$. It corresponds to the interpretation of matrix product as a composition of functions. This definition of matrix multiplication using paths in directed graphs may be also used in courses of discrete mathematics as an application of graph theory in linear algebra. It can be used in conjunction with the definition of matrix multiplication given in (Daugulis, Sondore, 2018).

EXAMPLE 5. Let $\boldsymbol{A}=\left[\begin{array}{ccc}a_{11} & a_{12} & 0 \\ 0 & a_{22} & a_{23}\end{array}\right], \boldsymbol{B}=\left[\begin{array}{cc}b_{11} & 0 \\ b_{21} & b_{22} \\ 0 & b_{32}\end{array}\right]$. Let us find the matrix product $\boldsymbol{A} \boldsymbol{B}$. It can be interpreted $\boldsymbol{A}$ and $\boldsymbol{B}$ as matrices of linear mappings. In the notations of the previous passage assume $B_{L}=\left[e_{1}, e_{2}\right]$, $B_{V}=\left[t_{1}, t_{2}, t_{3}\right], B_{U}=\left[z_{1}, z_{2}\right]$. The 2-LFGs on one picture are shown in Figure 4.

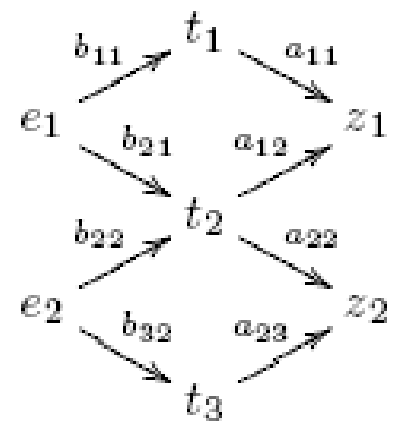

Figure 4. The description of $A B$ in terms of LFG for Example 5.

Looking at directed paths from the domain space to the range space the matrix $\mathrm{C}=\mathrm{AB}$ (Formula 4) can be established.

$$
\boldsymbol{C}=\boldsymbol{A} \boldsymbol{B}=\left[\begin{array}{cc}
b_{11} a_{11}+b_{21} a_{12} & b_{22} a_{12} \\
b_{21} a_{22} & b_{22} a_{22}+b_{32} a_{23}
\end{array}\right]
$$

For example, the element $c_{12}$ of matrix $C$ is equal to $b_{22} a_{12}$, because there is one directed path going from $e_{2}$ to $z_{1}$ path with edge weights $b_{22}$ and $a_{12}$.

\section{A new definition of determinant}

2-LFGs allow us to give a new definition of matrix determinant. Let $\boldsymbol{A}$ be a square matrix. Interpret it as the matrix of a linear mapping with respect to some basis $B$. Construct $\Lambda(B, B, A)$. It can be called its edge subset $P$ a permutation-edge-subset if

- $|P|=|B|$;

- every element of $B$ is the tail of some edge in $P$;

- $\quad$ every element of $B$ is the head of some edge in $P$.

Every permutation-edge-subset $P$ defines a permutation of $B$. Denote its sign by $\varepsilon(P)$. It is known that $\varepsilon(P)=(-1)^{c}$, where $c$ is the number of pairwise edge intersections for the standard plane embedding of the 2-FG of $P$ with straight edges. Denote $w(P)=\prod_{e \in P} w(e)$. Denote $s(P)=\varepsilon(P) \cdot w(P)$ and call it the signed edge weight product.

Comparing with the standard definition it is seen that $\operatorname{det}(\boldsymbol{A})=\sum_{P} S(P)$ where the summation is over all permutation-edge-subsets of $\Lambda(B, B, A)$.

EXAMPLE 6. Consider a general $3 \times 3$ matrix $A=\left[\begin{array}{lll}a_{11} & a_{12} & a_{13} \\ a_{21} & a_{22} & a_{23} \\ a_{31} & a_{32} & a_{33}\end{array}\right]$. It can be interpreted as the matrix of a linear operator with respect to some ordered basis $B=[1,2,3] . \Lambda(B, B, \boldsymbol{A})$ is shown in Figure 5 . 


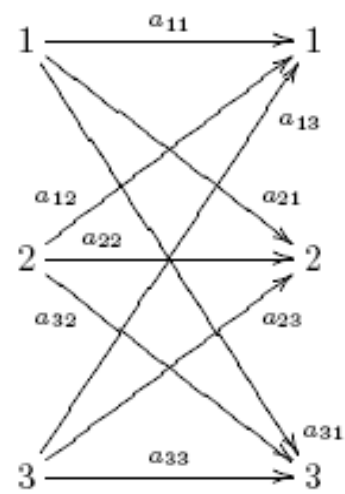

Figure 5. $\Lambda(\mathrm{B}, \mathrm{B}, \mathrm{A})$ of Example 6.

The number of permutation-edge-subsets is 6 , Figure 6 . The corresponding signed edge weight products (from left to right) are $a_{11} a_{22} a_{33},-a_{21} a_{12} a_{33},-a_{11} a_{32} a_{23},-a_{13} a_{22} a_{31}, a_{21} a_{32} a_{13}, a_{31} a_{12} a_{23}$.

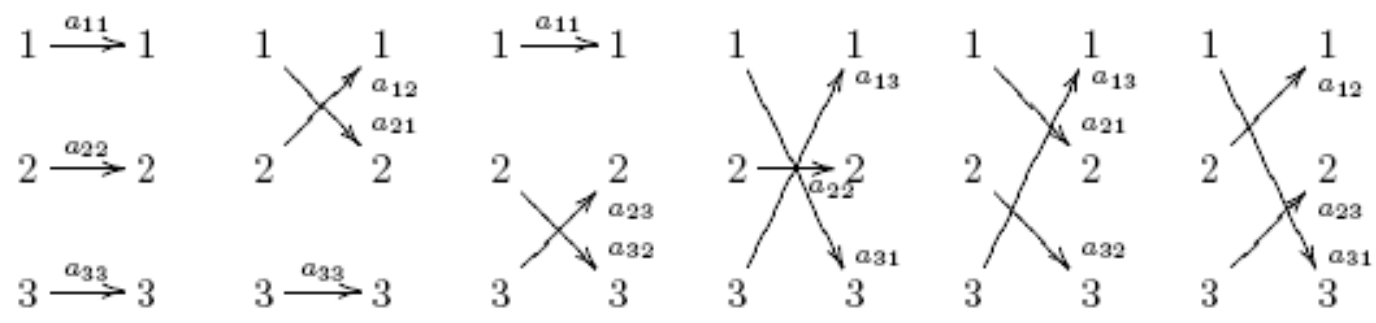

Figure 6 . The permutation-edge-subsets of $\Lambda(B, B, A)$ for Example 6 .

Summing over permutation-edge-subsets the authors get the determinant formula for $3 \times 3$ matrices -

$\operatorname{det}(\boldsymbol{A})=\sum_{P} s(P)=a_{11} a_{22} a_{33}-a_{21} a_{12} a_{33}-a_{11} a_{32} a_{23}-a_{13} a_{22} a_{31}+a_{21} a_{32} a_{13}+a_{31} a_{12} a_{23}$.

REMARK 3. Matrix elements corresponding to permutation-edge-subsets can be used to give yet another definition of determinant which does not involve LFG. Take a $n \times n$ matrix $\boldsymbol{A}$. For each permutation matrix $\boldsymbol{\Sigma}$ of the set $\{1, \ldots, n\}$ find the product of $\boldsymbol{A}$-elements which are in positions of 1's in $\Sigma$ and multiply it by the permutation sign $\varepsilon(\Sigma)$. Each such signed product cut out by a permutation matrix is equal to a signed edge weight product as defined above. Finally, sum these signed edge weights products over all permutations and get $\operatorname{det}(\boldsymbol{A})$.

\section{Graphs for linear operators}

Now the authors define linear functional graphs for linear operators using one basis. Let $L$ be a linear space with an ordered basis $B_{L}=\left[e_{1}, \ldots, e_{n}\right]$. Let a linear operator $f \in \operatorname{Hom}(L, L)$ be defined by the linear combination (5).

$$
f\left(e_{j}\right):=\sum_{i=1}^{n} f_{i j} e_{i}
$$

Again denote $\left[f_{i j}\right]$ - the $f$-matrix with respect to $B_{L}$ by $[f]_{B_{L}}$ or $\boldsymbol{F}$.

DEFINITION 2. Define a edge-weighted digraph $\Lambda\left(B_{L}, f\right)=\Lambda\left(B_{L}, \boldsymbol{F}\right):=\left(B_{L}, E, w_{f}\right)$, where $E=\bigsqcup_{i, j: f_{i j} \neq 0}\left(e_{i}, e_{j}\right)$. Edge weights are defined as follows: $w_{f}\left(e_{i}, e_{j}\right):=f_{i j} . \Lambda\left(B_{L} f\right)=\Lambda\left(B_{L}, \boldsymbol{F}\right)$ is called the 1-LFG of $f$ or $\boldsymbol{F}$ with respect to $B_{L}$.

REMARK 4. Note that $f(e)=0$ if and only if there is no edge left $e$ ( $e$ is a sink vertex). Vertices which are sinks or which have a loop and no outgoing edges are eigenvectors.

EXAMPLE 7. The 1-LFG for a Jordan block matrix $\left[\begin{array}{ccc}\lambda & 0 & 0 \\ 1 & \lambda & 0 \\ 0 & 1 & \lambda\end{array}\right]$ is shown in Figure 7. 


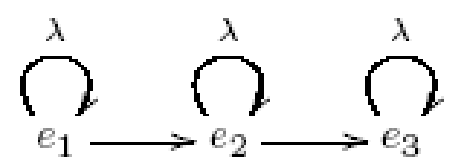

Figure 7. The 1-LFG for the Jordan block of Example 7.

\section{Using LFG in linear algebra courses}

LFG give opportunities to visualize linear mappings. Right plane embeddings (drawings) of LFG provide efficient arrangements of data. LFG can make linear algebra more accessible for visual learners. As it was shown LFG can be used to compute matrix products and determinants. Using LFG some linear algebra proofs, such as associativity of matrix multiplication and anti-homomorphism property of matrix transposition, are easier to see.

LFG has been used by the first author in university linear algebra classes, they often spark interest in students. It can contribute to developing research competencies of students (Kaskatayeva, 2014; Vintere, 2013). It should be noted that the introduction of LFG is more suitable at advanced levels rather than the first-year university courses.

\section{Conclusions}

We stress that the known matrix-digraph correspondence must be viewed as a generalization of functional graphs to the case of linear mappings and attention must be paid to the nature of vertices. The issue of graph vertices is not addressed properly in textbooks. Vertices of these graphs must be identified with the elements of bases. The task of finding a visual model for linear mappings has been addressed in the article and a solution has been described. This encoding of linear mappings and matrices as graphs is a helpful data arrangement and visualization technique which can be used teaching and studying linear algebra. This technique may expand visualization competence of students by giving another working example in a widely used and applied area of mathematics. Usage of graphs in other areas of mathematics can stimulate learning of graph theory and graphs models. In our opinion study courses and textbooks in linear algebra should be modified to include linear functional graphs as a desirable data arrangement and visualization technique for linear mappings.

Further discussions of LFG can involve interpreting basis changes in graphic terms. In a future development, functional graphs should also be generalized for other important algebraic structures such as groups.

\section{Bibliography}

1. Blyth T.S., Robertson E.F. (2002). Basic linear algebra (2nd edition). London: Springer Verlag London Limited.

2. Brualdi R.A., Cvetkovic D. (2009). A Combinatorial Approach to Matrix Theory and Its Applications. New York: Chapman and Hall/CRC. Retrieved from: https://www.routledge.com/A-CombinatorialApproach-to-Matrix-Theory-and-Its-Applications/Brualdi-Cvetkovic/p/book/9781420082234

3. Daugulis P. (1998). Stable endomorphism rings of idempotent E-modules (PhD thesis). University of Georgia, Athens, USA.

4. Daugulis P. (2012). A note on a generalization of eigenvector centrality for bipartite graphs and applications, Networks, 59(2), 261-264. doi: 10.1002/net.20442

5. Daugulis P., Krastiņa E., Sondore A., Vagale V. (2020). Skaitlisko datu izkārtošanas daudzveidība dzilāākai matemātikas izpratnei [Variety of arrangements of numerical data for a deeper understanding of mathematics]. In V. Lubkina, A. Indriksons (Eds.), The proceedings of the International Scientific Conference Society. Integration. Education. Rezekne: Rezekne Academy of Technologies, 107-118. doi: 10.17770/sie2020vol1.5081 (in Latvian)

6. Daugulis P., Sondore A. (2018). Visualizing matrix multiplication. PRIMUS, 28(1), 90-95. doi: 10.1080/10511970.2017.1313344

7. Doob M. (1984). Applications of Graph Theory in Linear Algebra. Mathematics Magazine, 57(2), 67-76. doi: 10.1080/0025570X.1984.11977080

8. Eurydice Brief, Modernisation of Higher Education in Europe: Access, Retention and Employability. (2014). Retrieved from: doi: 10.2797/61276 
9. Kalinina E. A., Khitrov G.M. (2017). A linear algebra approach to some problems of graph theory. In the Proceedings of International Conference on Computer Science and Information Technologies (CSIT), 11. Yerevan: IEEE, 5-8, doi: 10.1109/CSITechnol.2017.8312127

10. Kaskatayeva B. (2014). Pedagogical conditions for forming the research competency in future teachers of mathematics. In V. Dislere (Ed.), The Proceedings of the International Scientific Conference Rural Environment. Education. Personality (REEP), 7. Jelgava: LLU, 103-109. Retrieved from https://llufb.llu.lv/conference/REEP/2014/Latvia-Univ-Agricult-REEP2014proceedings-103-109.pdf

11. Kopeika E., Zvirgzdina L. (2020). Skills and competencies in mathematics of engineering students in context of sustainable development. In A. Aboltins (Ed.), The Proceedings of 19th International Scientific Conference Engineering for Rural Development, 19. Jelgava: Latvia University of Life Sciences and Technologies, 1255-1261. doi: 10.22616/ERDev.2020.19.TF303

12. König D. (1916). Über Graphen und ihre Anwendungen auf Determinanten-theorie und Mengenlehre [About graphs and their applications to determinant theory and set theory]. Mathematische Annalen, 77, 453-465. doi: 10.1007/BF01456961 (in German)

13. Li Y., Schoenfeld A.H. (2019). Problematizing teaching and learning mathematics as "given" in STEM education. International Journal of STEM Education (IJ STEM Ed), 6, 44. doi: 10.1186/s40594-019-0197-9

14. PISA 2021 Mathematics Framework. (2021). Retrieved from: https://pisa2021-maths.oecd.org/

15. Sergejeva N., Aboltins A. (2020). Knowledge of mathematics and physics as basis for studies in engineering sciences. In A. Aboltins (Ed.), The Proceedings of 19th International Scientific Conference Engineering for Rural Development, 19. Jelgava: Latvia University of Life Sciences and Technologies, 1302-1307. doi: 10.22616/ERDev.2020.19.TF325

16. Vintere A. (2013). Mathematics education process improvement through cooperation: experience of Latvia University of Agriculture. In V. Dislere (Ed.), The Proceedings of the International Scientific Conference Rural Environment. Education. Personality (REEP), 6. Jelgava: LLU, 354-361. Retrieved from https://llufb.llu.lv/conference/REEP/2013/Latvia-Univ-

Agricult_REEP_2013_ISSN_2255-808X-354-361.pdf

17. Zeidmane A., Rubina T. (2017). Causes of Failures in Mathematics by Engineering Students at Latvia University of Agriculture. In V. Dislere (Ed.), The Proceedings of the International Scientific Conference Rural Environment. Education. Personality (REEP), 10. Jelgava: LLU, 221228. Retrieved from https://llufb.llu.lv/conference/REEP/2017/Latvia-Univ-Agricult-REEP2017_proceedings-220-227.pdf

18. Zeidmane A., Rubina T. (2018). The Contribution of Mathematics to the Engineering Education in the Students' Assessment. In V. Dislere (Ed.), The Proceedings of the International Scientific Conference Rural Environment. Education. Personality (REEP), 11. Jelgava: Latvia University of Life Sciences and Technologies, 244-250. doi: 10.22616/REEP.2018.030

19. Zeidmane A., Sergejeva N. (2013). Indirect Impact of Mathematics in Engineering Education. In A. Aboltins (Ed.), The Proceedings of the Scientific International Conference Engineering for Rural Development.12. Jelgava: LLU, 611-615. Retrieved from http://www.tf.llu.lv/conference/proceedings2013/Papers/113_Zeidmane_A.pdf 